\title{
Análise da turbulência atmosférica na camada limite noturna em uma região de Floresta Amazônica
} Analisys of atmospheric turbulence in the nocturnal boundary layer over the
Amazon rain forest

Pablo Eli Soares Oliveira; Otávio Costa Acevedo; Felipe Denardin Costa; Daniel Michelon dos Santos; Claudio Alberto Teichrieb

Universidade Federal de Santa Maria; Universidade Federal do Pampa

pablo.deoliveira@gmail.com; otavio@ufsm.br; fdenardin@gmail.com; danielmichelon@gmail.com; teichrieb@gmail.com

\begin{abstract}
Resumo
Uma análise de dados de turbulência noturna, amostrados em diferentes níveis em uma região de floresta Amazônica, é feita para duas noites com comportamento distinto: uma na qual a turbulência foi contínua ao longo da noite, e outra em que a turbulência ocorreu de maneira intermitente. Para cada noite foram analisados, a partir da decomposição em multirresolução, os espectros de energia cinética turbulenta e velocidade vertical e coespectros dos fluxos de calor sensível.
\end{abstract}

Palavras-chave: Turbulência, Decomposição em multirresolução, Intermitência.

\begin{abstract}
In this study, turbulence data taken in different vertical levels in the Amazon rain forest are analyzed for two distinct nights: a night characterized by intense turbulent mixing, and a night which turbulence was intermittent. This is done using the multiresolution decomposition, which allows the identification of the turbulence and submeso contributions to spectra and cospectra.
\end{abstract}

Keywords: Turbulence, multiresolution decomposition, Intermittence. 


\section{Introdução}

Embora o método da covariância de vórtices seja amplamente utilizada para quantificar as trocas de quantidades, como energia, $\mathrm{CO}_{2}$ e momentum, entre as diferentes superfícies terrestres e a atmosfera, diversas dificuldades são encontradas no uso desta técnica, e em especial durante o período noturno, quando a intensidade da turbulencia diminui consideravelmente. Intermitência turbulenta ocorre em noites muito estáveis e é caracterizada por longos períodos calmos interrompidos por períodos de intensa atividade turbulenta (Coulter e Doran, 2002). Transportes que ocorrem em frequências mais baixas do movimento atmosférico, conhecidos como processos de submeso, podem se tornar importantes nessas condições. Estes transportes possuem uma grande variabilidade temporal, sendo influenciados por características locais, e tornam-se dominantes em condições na qual as escalas da turbulência são reduzidas, como em condições em que há um desacoplamento entre os diferentes níveis da camada limite noturna (Acevedo et al., 2014).

No presente trabalho, será feita uma análise de dados de turbulência atmosférica noturna em uma região de Floresta Amazônica, para duas noites com comportamento distinto: uma na qual a turbulência foi contínua ao longo da noite, e outra em que a turbulência ocorreu de maneira intermitente. O objetivo é identificar como as diferentes escalas do movimento interagem entre o interior da floresta e o ar acima desta.

\section{Fundamentação teórica e métodos}

\subsection{Intermitência}

Em uma camada limite fortemente estratificada, a turbulência pode ocorrer, muitas vezes, em eventos esporádicos de curto tempo de vida, em um fenômeno conhecido como intermitência global (Mahrt, 1999). Eventos de turbulência intermitente são favorecidos em condições muito estáveis, em noites de céu claro e vento fraco, ou com advecção de ar quente sobre uma superfície mais fria, em que longos períodos calmos são eventualmente interrompidos por períodos de intensa atividade turbulenta (Coulter e Doran, 2002). Estes trazem grandes complicações na determinação do balanço dos fluxos turbulentos na camada limite noturna devido ao seu caráter aproximadamente aleatório. Durante estes eventos, o período normalmente utilizado para o cálculo dos fluxos turbulentos pelo método clássico da covariância de vórtices pode não ser adequado. Transportes de baixa frequência, cujas estimativas são bem mais imprecisas, podem aparecer na estimativa de meia hora, aumentando significativamente o erro associado à medida. Acevedo et al. (2006) mostraram que eventos intermitentes possuem diterentes escalas de tempo, tazendo com que os fluxos noturnos sejam extremamente dependentes da janela de tempo utilizada. Oliveira et al. (2013) mostraram que em eventos intermitentes intensos, os grandes fluxos são causados pelas escalas de baixa frequência do movimento turbulento. Ainda, estes fluxos não dependem apenas da intensidade da turbulência, mas também de como ela evolui ao longo do tempo.

\subsection{Fator de Intermitência}

Neste trabalho, a quantificação da intermitência é feita por um método similar ao utilizado por Coulter e Doran (2002), que consiste em uma maneira de estimar se a noite foi turbulenta ou intermitente através dos fluxos superficiais. Os fluxos de calor sensível foram calculados, para cada noite, a cada 5 minutos, e classificados em ordem decrescente. O fator de intermitência (FI) é dado pela fração de tempo necessária para atingir a metade do fluxo noturno total nas séries ordenadas. Se a turbulência for contínua, esta fração será 0,5 , que é, portanto, o limite máximo do fator de intermitência, e característico de turbulência plenamente desenvolvida. Para turbulência intermitente, valores menores de fator de intermitência ocorrem. A figura 1 mostra a fração cumulativa normalizada do fluxo noturno de calor sensível como função da fração do tempo total e a evolução temporal da velocidade vertical para a noite mais turbulenta e uma das noites mais intermitentes. Os valores de fator de intermitência encontrados neste caso foram 0,09 e 0,31 para a noite intermitente e turbulenta, respectivamente.
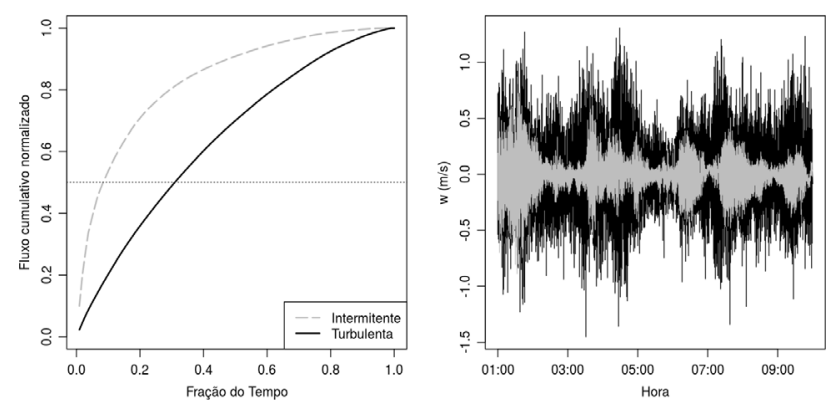

Figura 1: Fração cumulativa normalizada do fluxo noturno de calor sensível como função da fração do tempo total e evolução temporal da velocidade vertical, para uma noite intermitente (linha cinza) e para uma noite turbulenta (linha preta).

\subsection{Decomposição em multirresolução}

Na decomposição em multirresolução, a série turbulenta é decomposta em suas escalas temporais. Calcular as médias da série temporal usando diferentes comprimentos 
médios é equivalente a visualizar os dados em diferentes resoluções. Espectros e coespectros da multirresolução podem ser interpretados em termos de médias móveis não ponderadas (Vickers e Mahrt, 2003), no método da transformada de Haar (Howell e Mahrt, 1997) e em termos de ondeletas (Mallat, 1989).

A decomposição em multirresolução satisfaz as propriedades das médias de Reynolds, e a integração do espectro $S$ da decomposição em multirresolução até uma escala de tempo $\tau$ é igual a variância da série original de comprimento $\tau$. Desta forma, para o espectro de uma série com $2^{p}$ pontos de dados de uma quantidade $\phi$, com $p \geq M$, tem-se que:

$$
\sum_{n=1}^{M}\left(S_{\phi}\right)_{n}=\left\langle\overline{\phi^{\prime 2}}\right\rangle_{\tau}
$$

E, da mesma forma para a covariância, tem-se:

$$
\sum_{n=1}^{M}\left(S_{w \phi}\right)_{n}=\left\langle\overline{w^{\prime} \phi^{\prime}}\right\rangle_{\tau}
$$

Onde $\tau=\frac{2^{M}}{f}$ e f é a frequência de amostragem.

\section{Sítio experimental e dados utiliza- dos}

A torre micrometeorológica está localizada na Reserva de Desenvolvimento Sustentável Uatumã, $\left(2^{\circ} 08.602^{\prime} \mathrm{S}\right.$, $\left.59^{\circ} 00.033^{\prime} \mathrm{O}\right)$. A floresta possui uma altura média de $37 \mathrm{~m}$, e torre possui $81 \mathrm{~m}$ de altura, na qual são feitas diversas medidas meteorológicas. Informações detalhadas sobre o sítio experimental e instrumentos utilizados podem ser obtidas em Andreae et al. (2015).

Foram utilizados dados noturnos, entre 21 e 06 horas (Hora Local) de velocidade do vento e temperatura registrados a uma frequência de $10 \mathrm{~Hz}$ nos níveis de 04 , 36, 46 e $81 \mathrm{~m}$, utilizando um anemômetro sônico 3-D (Windmaster, Gill Intruments). Ao todo, foram analisadas 150 noites de dados, do dia 01 de janeiro até 30 de maio de 2013. Entretanto, nem todos os níveis estavam disponíveis durante todas as noites, sendo utilizados apenas as noites na qual havia registro em todos os níveis, totalizando, efetivamente, 114 noites. Espectros e coespectros de multirresolução foram calculados para séries de $2^{16}$ pontos, em torno de 109 minutos, com passos de 30 minutos, gerando um total de 15 séries para cada noite. Este procedimento cria uma certa redundância, de modo que uma dada porção do tempo é analisada em diferentes séries subsequentes. A razão para isto é maximizar a chance de que eventos intermitentes específicos sejam devidamente capturados em uma dada série temporal.

O fator de intermitência foi calculado para o fluxo de calor sensível, no nível de $46 \mathrm{~m}$, para todas as noites, e a noite mais turbulenta e uma das noites mais intermitentes (ambas determinadas pelo fator de intermitência) foram utilizadas para análise.

\section{Resultados}

Espectros da velocidade vertical e energia cinética turbulenta, e coespectros dos fluxos de calor sensível são apresentados para duas noites distintas: a que apresentou fator de intermitência mais alto, que corresponde a uma noite com turbulência bem desenvolvida, e uma com fator de intermitência baixo, correspondendo a uma noite em que a turbulência ocorreu de maneira intermitente. Não foi utilizada a noite com menor fator de intermitência pois essa apresentou apenas um evento extremamente intenso de aumento da turbulência que foi responsável pela diminuição do fator de intermitência. As séries temporais da velocidade vertical em $46 \mathrm{~m}$ dessas noites foram mostradas na figura 1, juntamente com o fluxo cumulativo normalizado da qual se obtém o fator de intermitência. A noite turbulenta, com maior valor do fator de intermitência, teve turbulência intensa ao longo de toda a noite com grande parte das flutuações acima de $0.4 \mathrm{~ms}^{-1}$, e com máximos chegando a $1 \mathrm{~ms}^{-1}$ em 36 e 46 e $81 \mathrm{~m}$. Na noite intermitente, a intensidade da turbulência foi menor, com as flutuações máximas chegando a $0.5 \mathrm{~ms}^{-1}$, mas com a alternância de períodos calmos e períodos de atividade turbulenta durante toda a noite.

Os espectros de energia cinética turbulenta mostram, na noite intermitente, a grande contribuição dos transportes de mais baixa frequência nas maiores escalas temporais, nos níveis de 36, 46 e $81 \mathrm{~m}$ (Figura 2). Na noite turbulenta, essa contribuição é menor e se torna menos relevante que a contribuição de processos turbulentos nos níveis próximos ao topo da copa. A escala de tempo na qual os processos de submeso começam a ser observados nos espectros é menor na noite intermitente do que na noite turbulenta, e diminui com a altura, indo de 100 segundos em $36 \mathrm{~m}$ para pouco mais de 10 segundos em $81 \mathrm{~m}$. A partir desse ponto, há um aumento acentuado da energia cinética turbulenta com o aumento da escala temporal, e essa diferença é maior do que a observada nos espectros médios. Acevedo et al. (2014) notaram que há uma relação exponencial entre a energia cinética turbulenta e a escala temporal, e que esta relação é altamente dependente de $\sigma_{w}$, com maiores expoentes para condições menos turbulentas, do mesmo modo que o observado aqui. Em 46 e $81 \mathrm{~m}$, somente a noite turbulenta apresenta uma região turbulenta bem definida nos espectros, com o segundo sendo novamente o nível mais energético nas maiores escalas temporais e na qual a energia cinética turbulenta independe do comportamento da turbulência ao longo da noite. Já em 
$4 \mathrm{~m}$, a energia está concentrada em escalas temporais longas, e é mais intensa na noite intermitente que na noite turbulenta. Adicionalmente, a noite intermitente tem uma maior contribuição relativa em submeso acima da copa. Isto reforça a ideia de que a energia cinética turbulenta nos níveis mais baixos da floresta é mais afetada por processos de submeso acima da floresta do que pela atividade turbulenta nestes níveis.
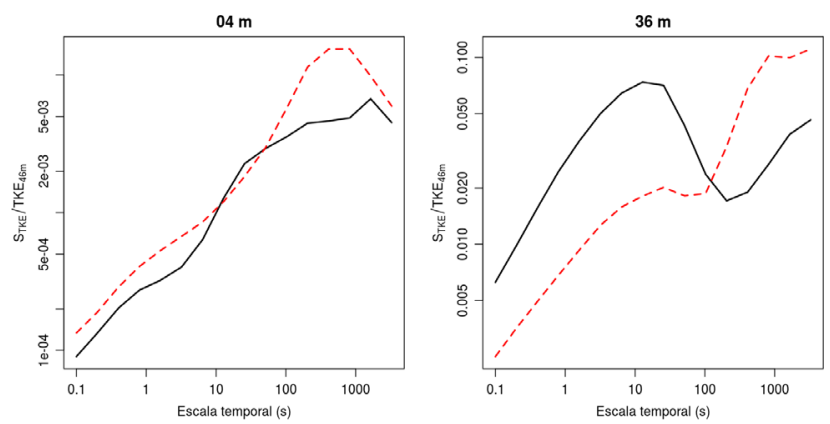

$46 \mathrm{~m}$
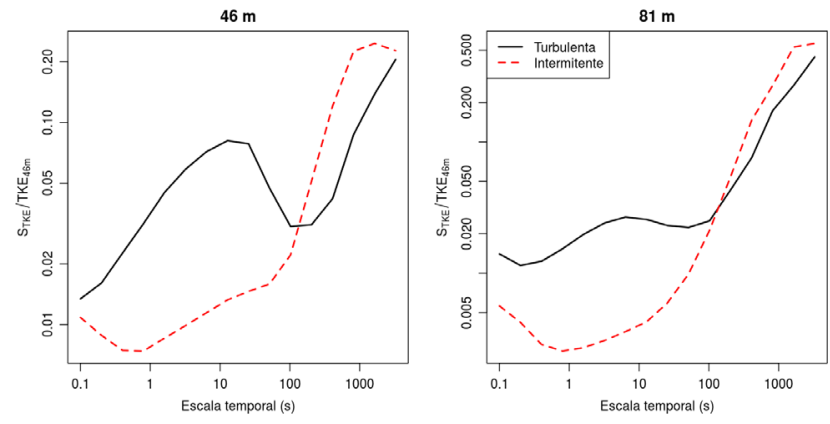

Figura 2: Espectros de multirresolução da energia cinética turbulenta (TKE) normalizada pela energia cinética turbulenta em $46 \mathrm{~m}$ para uma noite com turbulência intensa e para uma noite com turbulência intermitente.

Os espectros da velocidade vertical mostram claramente uma dependência com o comportamento da turbulência (Figura 3). Os picos espectrais ocorrem em menores escalas temporais para a noite turbulenta, e são de difícil determinação nos níveis superiores para a noite intermitente. $\mathrm{O}$ nível inferior mostra novamente um resultado interessante. Além dos picos espectrais ocorrerem em escalas temporais muito maiores do que nos níveis mais altos, possivelmente devido à atenuação de movimentos de alta frequência pelas folhas (Fitzjarrald e Moore, 1990), a relação entre a intensidade da turbulência acima e no interior da floresta está invertida. A variância da velocidade vertical, dada pela soma dos espectros em todas as escalas de tempo, é maior na noite intermitente, que é mais afetada por transportes de baixa frequência, do que na noite turbulenta, menos afetada, e isso é o oposto do que é observado nos demais níveis. Isso mostra que, do mesmo modo que ocorre para TKE, os processos turbulentos dentro da floresta podem ser controlados por processos de baixa frequência fora dela.
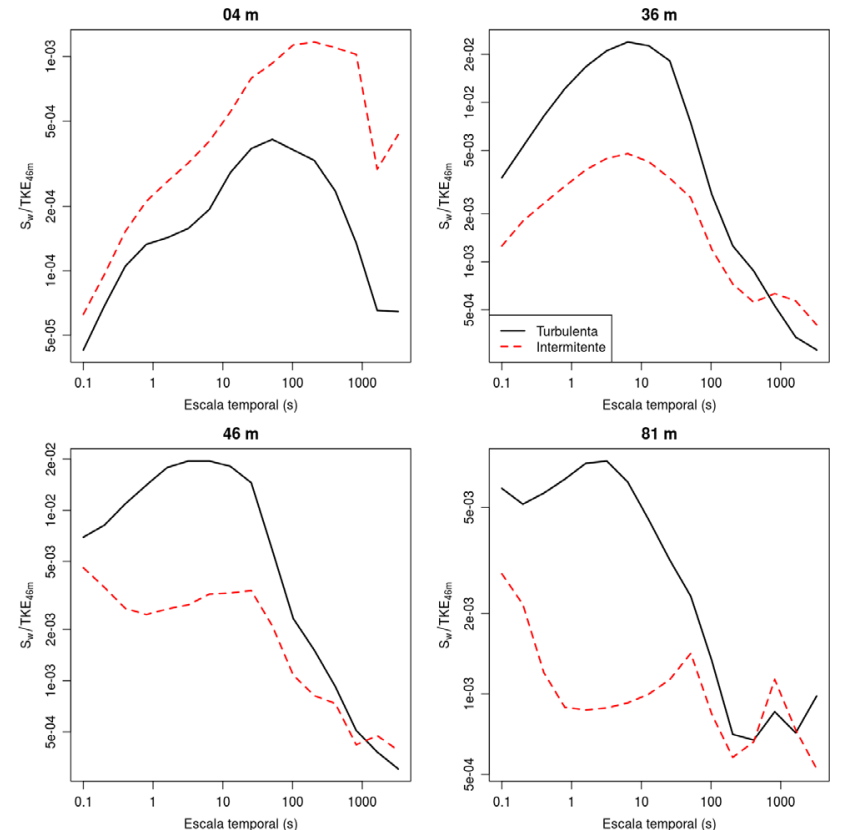

Figura 3: Espectros de multirresolução da velocidade vertical normalizados pela energia cinética turbulenta (TKE) em 46 m para uma noite com turbulência intensa e para uma noite com turbulência intermitente.

Já para os coespectros dos fluxos de calor sensível, a diferença entre a noite turbulenta e a intermitente se acentua (Figura 4). Os coespectros parecem ser altamente influenciados pelos transportes de baixa frequência em todos os níveis na noite intermitente. Enquanto que para a noite turbulenta, os coespectros tem uma região turbulenta bem definida, para a noite intermitente eles são erráticos desde as menores escalas temporais, com uma sobreposição dos processos de submeso sobre os processos turbulentos que faz com que a escala temporal dos picos e das falhas coespectrais não seja facilmente determinada.

O nível de $04 \mathrm{~m}$ é novamente o que mais chama a atenção. Os fluxos de calor sensível são maiores na noite intermitente do que na turbulenta, o oposto do que ocorre nos outros níveis (em valores absolutos). Isso favorece um maior resfriamento no interior da floresta e um aquecimento logo acima dela, como mostra a figura 5, já que a energia é transferida dos níveis mais baixos para o topo. Na noite turbulenta, a temperatura cai mais rapidamente nos níveis mais altos do que em $04 \mathrm{~m}$, principalmente nas primeiras horas da noite. Entretanto, as camadas tendem a se resfriar uniformemente, e os perfis mantêm a mesma forma ao longo da noite. $\mathrm{Na}$ noite intermitente, há um resfriamento mais intenso no nível inferior do que acima da floresta quando a turbulência diminui, como pode-se observar em 02-03, 03-04 e 09-10, e mais intenso também com relação ao que se 

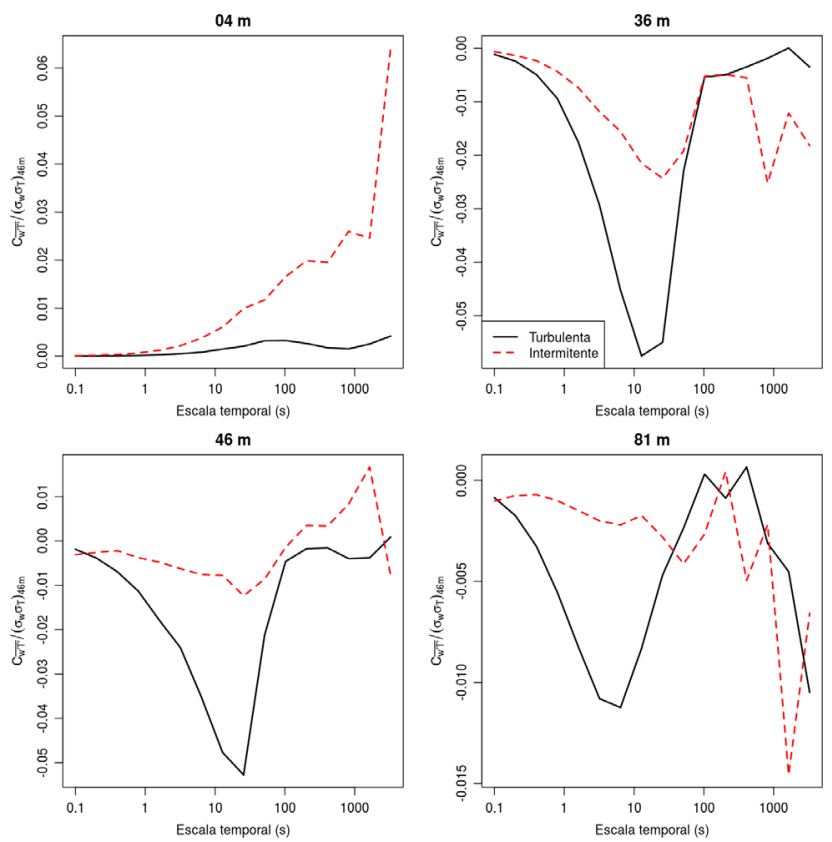

Figura 4: Coespectros de multirresolução dos fluxos de calor sensível normalizados pelo desvio padrão da temperatura multiplicado pelo desvio padrão da velocidade vertical em $46 \mathrm{~m}$ para uma noite com turbulência intensa e para uma noite com turbulência intermitente.

observa na noite turbulenta. A queda mais acentuada ocorre no perfil 03-04, na qual uma condição mais turbulenta sucede uma condição de calmaria. O nível de 46 m não mostra queda de temperatura maior do que alguns poucos décimos de grau ao longo da noite. Inclusive, acaba ocorrendo o contrário em algumas horas, já que a maior temperatura nesse nível ocorre no perfil 09-10, juntamente com a menor temperatura observada no nível mais baixo.

Isso mostra que a estratificação térmica, que atenua a produção de turbulência em condições estáveis, tende a aumentar em condições de turbulência intermitente, o que pode levar a uma diminuição ainda maior da turbulência no interior da floresta. Aqui, o gradiente de temperatura é maior na noite turbulenta nas primeiras horas, mas a figura 5 mostra que a velocidade do vento acima da floresta também foi muito maior do que a observada na noite intermitente, levando a uma produção maior de turbulência por cisalhamento do vento.

\section{Conclusões}

Neste trabalho, foi feita uma análise de dados de turbulência de duas noites com características distintas, amostrados em diferentes níveis em uma região de Floresta Amazônica. Espectros da energia cinética turbulenta e
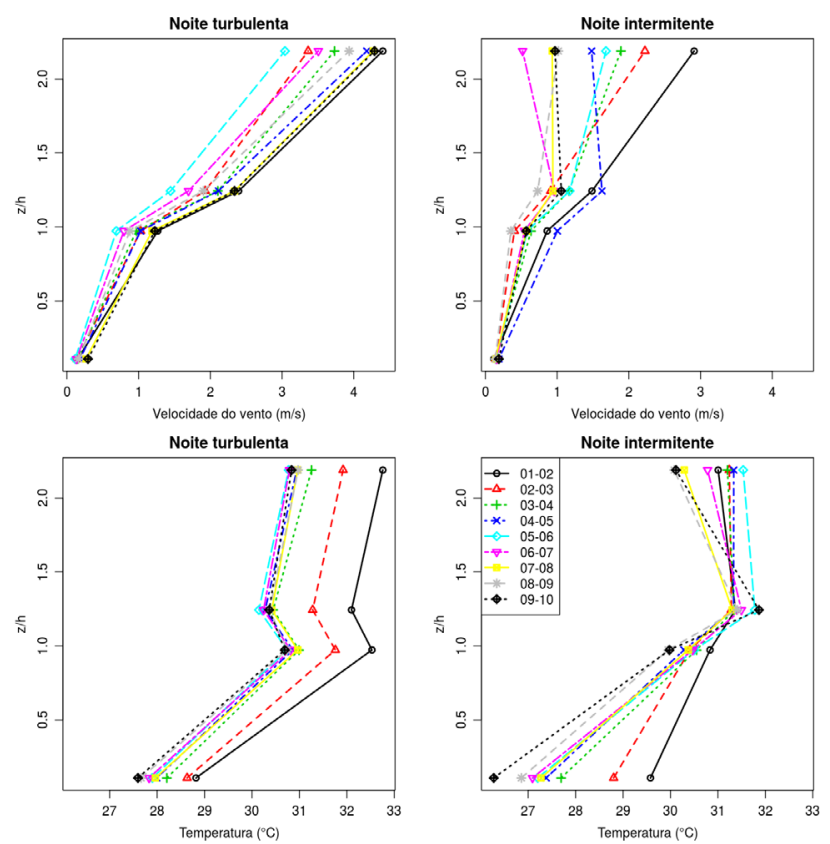

Figura 5: Perfil médio de velocidade do vento e temperatura para a noite turbulenta (painéis à esquerda) e para a noite intermitente (painéis à direita). Cada linha representa a temperatura média em uma hora (GMT), dada pela legenda.

da velocidade vertical e coespectros dos fluxos de calor sensível foram analisados, focando nos problemas que ocorrem em noites com turbulência intermitente, comparando-as com casos em que a turbulência é bem desenvolvida.

É evidente que os processos de submeso influenciam o comportamento dos transportes noturnos, e que esses processos se tornam mais importantes com o aumento da intermitência. Os resultados indicam que há mais atividade turbulenta junto à superfície (04 m) nos casos intermitentes que em casos de turbulência bem desenvolvida, uma inversão em relação ao que ocorre nos demais níveis. Esse contraste foi maior para o fluxo de calor sensível, de forma que há indícios que este resultado tenha grande influência nas transferências de escalares dos níveis inferiores da copa para seu topo e a atmosfera acima. Isso fica mais evidenciado pelo impacto mostrado nos perfis de temperatura.

\section{Referências}

Acevedo, O. C., Moraes, O. L. L., Degrazia, G. A., Medeiros, L. E. (2006). Intermittency and the exchange of scalars in the nocturnal surface layer. Boundary-Layer Meteorology, 119, 41-55.

Acevedo, O. C., Costa, F. D., Oliveira, P. E. S., Puha- 
les, F. S., Degrazia, G. A., Roberti, D. R. (2014). The influence of submeso processes on stable boundary layer similarity relationships. Journal of the Atmospheric Sciences, 71, 207-224.

Andreae, M. O., Acevedo, O. C., Araùjo, A., Artaxo, P., Barbosa, C. G. G., Barbosa, H. M. J., Brito, J., Carbone, S., Chi, X., Cintra, B. B. L., da Silva, N. F., Dias, N. L., Dias-Júnior, C. Q., Ditas, F., Ditz, R., Godoi, A. F. L., Godoi, R. H. M., Heimann, M., Hoffmann, T., Kesselmeier, J., Könemann, T., Krüger, M. L., Lavric, J. V., Manzi, A. O., Moran-Zuloaga, D., Nölscher, A. C., Nogueira, D. S., Piedade, M. T. F., Pöhlker, C., Pöschl, U., Rizzo, L. V., Ro, C. U., Ruckteschler, N., Sá, L. D. A., Sá, M. D. O., Santos, C. B. S. M. N. D., Saturno, J., Schöngart, J., Sörgel, M., de Souza, C. M., de Souza, R. A. F., Su, H., Targhetta, N., Tóta, J., Trebs, I., Trumbore, S., van Eijck, A., Walter, D., Wang, Z., Weber, B., Williams, J., J. Winderlich, S. W., F. Wittmann, Yáñez-Serrano, A. M. (2015). The amazon tall tower observatory (atto) in the remote amazon basin: overview of first results from ecosystem ecology, meteorology, trace gas, and aerosol measurements. Atmospheric Chemistry and Physics, 15, 11,599 - 11,726.

Coulter, R. L., Doran, J. C. (2002). Spacial and temporal occurrences of intermittent turbulence during cases-99. Boundary-Layer Meteorology, 105, 329-349.

Fitzjarrald, D. R., Moore, K. E. (1990). Mechanisms of nocturnal exchange between the rain forest and the atmosphere. Journal of Geophysical Research, 95, 16,83916,850 .

Howell, J. F., Mahrt, L. (1997). Multiresolution flux decomposition. Boundary-Layer Meteorology, 83, 117-137.

Mahrt, L. (1999). Stratified atmospheric boundary layers. Boundary-Layer Meteorology, 90, 375-396.

Mallat, S. (1989). A theory for multiresolution signal decomposition: The wavelet representation. IEEE Trans Pattern Anal Machine Intell, 11(7), 674-693.

Oliveira, P. E. S., Acevedo, O. C., de Moraes, O. L. L., Zimmermann, H. R., Teichrieb, C. A. (2013). Nocturnal intermittent coupling between the interior of a pine forest and the air above it. Boundary-Layer Meteorology, $146,45-64$.

Vickers, D., Mahrt, L. (2003). The cospectral gap and turbulent flux calculations. Journal of atmospheric and oceanic technology, 20, 660-672. 